\title{
Prefrontal abilities
}

\author{
D.F. Benson
}

UCLA School of Medicine, 710 Westwood Plaza, Los Angeles, CA 90024-1769, USA

The neuroanatomical region that has most prominently altered with the advancing cognitive competency of the human is the prefrontal cortex, particularly the rostral extreme. While the prefrontal cortex does not appear to contain the neural networks that carry out cognitive activities, the management of these high level manipulations, so uniquely characteristic of the human, appears dependent upon the prefrontal cortex.

Keywords: Heteromodal association cortex - Prefrontal cortex - Supramodal association cortex - Unimodal association cortex

\section{INTRODUCTION}

It is generally acknowledged that the human has the capacity to perform more complex, high level cognitive manipulations than do other animals and that the brain is the site of this activity (Young, 1987). A number of differences in structure distinguish human brains from those of other animals and these unique structures apparently participate in the human's ability to "think". One of these brain regions, the frontal cortex, will be given particular attention in this essay. Human frontal lobes are both larger and more obvious than those of other animal species, a prominence that led early investigators to suggest that they are the seat of human intelligence (Bianchi, 1895; Tilney, 1928). Many correlations of brain and behavior in the human stress frontal lobe functions.

Factual data to support this seductive correlation have proved hard to develop and whether the high level intellectual and moral functions said to characterize human behavior can truly be consigned to frontal functions has been heatedly debated (Franz, 1907; Feuchtwanger, 1923; Rylander, 1939; Goldstein, 1944; Hebb, 1945; Halstead, 1947; Mettler, 1949; Weinstein and Teuber, 1957; Teuber, 1959). A number of investigators (Lashley, 1929; Chapman and Wolff, 1959) insisted that the higher level of mental function in humans reflected only greater brain mass, not development within any single brain area. Proof that human moral and intellectual capabilities are products of the evolution of the human frontal lobes is not readily observed.

Two problems were obvious. First, factual data to demonstrate that evolutionary changes have occurred in the structure of the human brain have not been available (Kotchetkova, 1978; Jerison, 1991). A second, equally troublesome, point is that little direct evidence has been developed to indicate what part, if any, the frontal cortex plays in the advanced mental functions of humans.
Attempts to explore whether, and in what manner, the magnificent changes in cognitive activity currently enjoyed by humans have involved the frontal lobes, produce intriguing questions. Has there been any actual change (evolution) in the anatomical structures of the frontal cortex? If so, can they be considered the seat of the behavioral advances? Are the revolutionary advances in "technological" competency so notable over the past two centuries related to changes in brain structure/function or do they stem from improved use of pre-existing brain capabilities? Investigation of these questions demands separate examination of the evolution of the human brain and of the evolution of human behavior.

\section{ANATOMICAL EVOLUTION}

The anatomical structure of the human brain shows a number of differences when compared with brains of other animal species. Obvious features include its relatively greater size and some notable alterations of brain configuration.

Considering the size differential as a question of weight alone, the human brain ranks as one of the heaviest (largest) of all animal species (see the first column of Table I). Only a few currently existing animal species have larger brains than the human and in these the brain/body weight ratio (column 2) is considerably below that of the human. Based on brain weight to total body weight ratio, the human brain appears to have more potential to develop complex, non-reflex neural activities. The size differences are not enormous, however; size alone does not appear to be responsible for the considerable behavioral differences between man and other animals.

Less obvious but no less significant, the increased brain size of the human does not represent equal growth of all brain structures. Selected regions of human brain have 
TABLE I. Brain size

\begin{tabular}{lcc}
\hline & Brain weight $(g)$ & $\begin{array}{c}\text { Brain weight: } \\
\text { body weight ratio }\end{array}$ \\
\hline Whale & 6800 & $1: 854$ \\
Elephant & 4717 & $1: 646$ \\
Dolphin & 1735 & $1: 82$ \\
Man & 1444 & $1: 44$ \\
Walrus & 1126 & $1: 592$ \\
Camel & 762 & $1: 525$ \\
Giraffe & 680 & $1: 778$ \\
Hippopotamus & 582 & $1: 3015$ \\
Horse & 532 & $1: 692$ \\
Polar bear & 498 & $1: 519$ \\
Ox & 493 & $1: 1339$ \\
Chimpanzee & 440 & $1: 128$ \\
\hline
\end{tabular}

(Adapted from Blinkov and Glezer, 1968, and Cook, 1986).

enlarged considerably in comparison to these regions in the brains of other species. The difference is most notable in the cortex, the thin mantle of neural cells that overlies the brain surface of all higher species. The cortical gyri and sulci are far more complex in the human than in other mammalian species, greatly increasing the extent of cortical surface. Some of the complex gyral patterns appear mechanical, designed to fit the increasingly large cortical surface into a finite, fixed container, the skull. Brain gyration is not entirely mechanical, however, and correlation between increased gyral complexity and the development of high level behavior is apparent. Thus, while total volume and weight of human brain are somewhat greater than in other species, an inordinate percentage of this increase represents cortical surface.

The increase in human cortical surface is not uniform. Three areas in particular have shown considerable growth: (1) prefrontal cortex; (2) inferior parietal cortex (angular gyrus); (3) middle temporal gyrus. All three areas are recognized as significant for higher level mental processing (Benson, 1993). Functions ascribed to the angular gyrus and to the second temporal gyrus are discussed elsewhere in this volume. This paper will explore the significance of the considerable enlargement of the human prefrontal cortex.

Whether the prefrontal cortex has actually changed (evolved) in the span of human existence remains unknown and debatable (Hodos, 1970; Campbell, 1975; Kotchetkova, 1978). There can be no doubt, however, that brain changes have occurred through zoological evolution, at least as indicated by comparison with existing species. Figure 1, taken from the seminal studies of the prefrontal cortex by Fuster $(1980,1989)$, illustrates the increasing size of the prefrontal cortex in six well-studied mammalian species, differences that are sufficient to be recognizable even in simple illustration. Brodmann (1912) estimated that the "regiofrontalis", the basic pre- frontal cortex, occupied $29 \%$ of total cortical area in the human, $17 \%$ of the cortex of the chimpanzee, $11.5 \%$ in the gibbon and macaque, and $8.5 \%$ in the lemur. In the dog and cat, similar measurements are $7 \%$ and $3.5 \%$, respectively, and the numbers become disappearingly small in rodents. When the considerably larger relative size of the prefrontal cortex is combined with the significantly larger brain, the human prefrontal cortex is tremendously larger than that of other animal species. While not unique to the human, the prefrontal cortex is both more extensive and better developed in the human. It would appear reasonable that this development has led to behavioral changes, including the technological advances that characterize current society.

\section{BEHAVIORAL EVOLUTION}

Charting alterations of human behavior over time has proved just as difficult as categorizing anatomical differences. Most studies of ancient behavior, the field of anthropology, rely on discovery and interpretation of artefacts, the tools used for everyday life. The technological revolution of the past century would prove impossible to chart by this crude method and it appears probable that the picture provided of earlier cultures may be similarly inadequate. A second approach is the study of primitive cultures, the lost tribes of jungle recesses, discovered and investigated by explorers. While these studies provide valuable insights, they focus on the basic life patterns developed to maintain existence within the milieu. Little conjecture (and even less investigation) has been formulated concerning differences, if any, in the brain structure of these primitive individuals that can be correlated with the behavioral characteristics demanded by distinct life situations. Most anthropological studies of primitive cultures lack descriptions of high level behaviors, and when such behaviors have been described (e.g. spoken language patterns) they lack correlation with other brain functions or with neuroanatomy. Whether minor alterations of brain structure accompany the diversity of cultural patterns of isolated tribes has never been contemplated, much less studied.

One prime reason for the dearth of information on the primitive human's behavior, particularly the correlation of prefrontal anatomical development with behavioral idiosyncrasies, stems from the crudely primitive level of current understanding of frontal influences on behavior. Frontal lobe function remained totally mysterious well into the 20th century (Young, 1970). While an important role for prefrontal structures was suspected, even emphasized, from the latter part of the 19th century (Munk, 1890; Bianchi, 1895) and active research was performed by many investigators early in the 20th century (Grünbaum and Sherrington, 1903; Franz, 1907; Tilney, 1928; Fulton, 

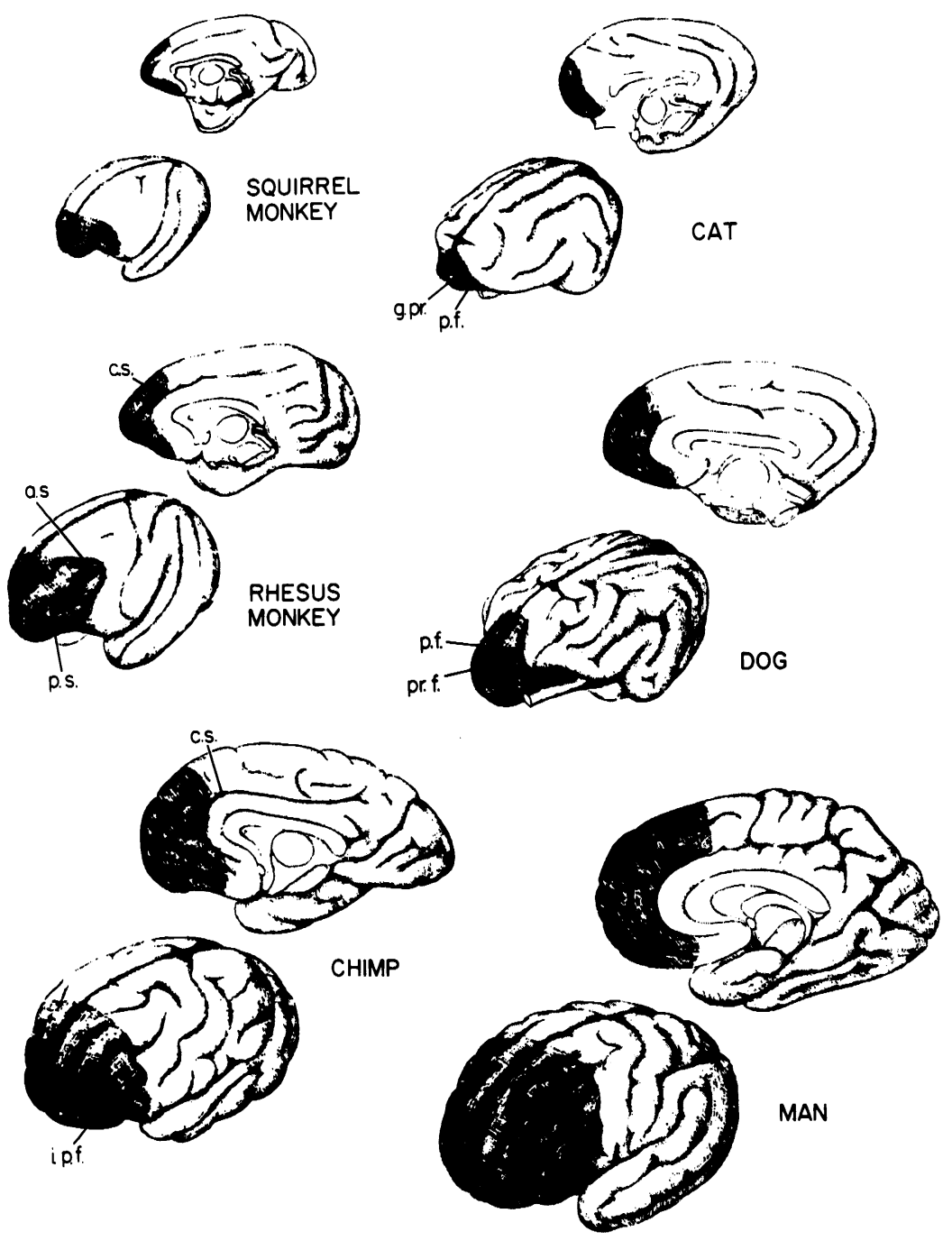

FIG. 1. The prefrontal cortex (marked by shading) in six different species. Abbreviations: a.s., arcuate sulcus; c.s., cingulate sulcus; g.pr., gyrus proreus; i.p.f., inferior precentral fissure; p.f., presylvian fissure; p.s. principal sulcus; pr.f., proreal fissure. (From Fuster, 1989, with permission.)

1952), reliable data remained sparse and the frontal lobes remained a riddle (Teuber, 1964). Pertinent experiments were performed but correlation of laboratory observations on animal behavior with human behavior proved disappointing. One problem confounding the early investigations of frontal function stemmed from the ingrained acceptance of the reflex arc as basic to nervous system physiology. Frontal functions did not fit this scheme.

In the 1960s, Pribram and colleagues (Miller et al., 1960; Pribram, 1960) contended that the reflex arc was inappropriate for understanding frontal lobe functions. A feedback loop that involved prefrontal structures was proposed, a test-operate-test-exit (TOTE) operation (Miller $e t$ al., 1960). The postulation of a testing (monitoring) func- tion proposed for prefrontal cortex represented a new concept. Other investigators (Teuber, 1964; Nauta, 1971; Luria, 1973) verified and expanded this thesis. Anatomists (Fuster, 1980, 1989; Pandya et al., 1981; Goldman-Rakic, 1990) and behaviorally oriented investigators (Shallice and Evans, 1978; Shallice, 1982; Damasio, 1985; Stuss and Benson, 1986; Goldberg and Bilder, 1987) have produced theories of frontal function based on neuropsychological testing, animal lesion studies, and clinical observations. The growing collection of ideas, observations and experimental data has generated new concepts of frontal lobe function.

Luria (1973) posited three major brain activities-cerebral tone, sensory-motor processing, and mental regu- 
lation-and suggested that brain stem and diencephalic structures performed the first function, the posterior cortex the second, and the prefrontal cortex the third. Fuster $(1980,1989)$ demonstrated that prefrontal structures managed serial stimuli over time, providing a temporal gradient for mental processing. Damasio (1985) emphasized the ability of frontal structures to manage multitudes of stimuli, a gating effect. Shallice (Shallice and Evans, 1978; Shallice, 1982) theorized four major cognitive functions, calling them: (1) cognitive units; (2) schemas; (3) contention scheduling; (4) supervisory attentional systems. The four components indicated the increasing interaction needed to perform higher level mental functions. Only his fourth level - the ability to handle non-routine, goal-directed activities-demanded prefrontal function. Stuss and Benson (1986) proposed a hierarchical scheme of brain function. They noted that all basic psychological activities (arousal, attention, emotion, sensory, motor, visual imagery, memory, language, and cognitive manipulation) were performed by basal and/or posterior brain structures. In this model, prefrontal functions (drive, sequencing, executive control, self-analysis) were "supramodal" controlling mechanisms that acted through the nine basic functions (see Fig. 2). Major prefrontal functions included the ability to activate (motivate) behavioral responses (drive), to maintain serial order (sequencing), to monitor and manage the basic psychological functions (executive control), and to ruminate about past behaviors and to contemplate future activities (self-analysis).

Descriptions of prefrontal function have developed too rapidly in the past several decades to provide a stable behavioral base for correlation with neuroanatomy, much less investigate the relationship of prefrontal functions and brain evolution. Little firm evidence, either for or against, indicates change in human prefrontal competency. That development of more advanced brain structures has produced the superior emotional control and intellectually guided technological development of the human is an enticing, but as yet unproved, hypothesis.

\section{THEORIES OF COGNITIVE EVOLUTION}

Vast changes in the behavioral capabilities of the human have occurred in a rapidly, almost geometrically expanding manner over the past several centuries. Are there anatomical alterations in the brain to account for any of these changes? Has neuroanatomical evolution provided the base for the current technological revolution? Three theories will be considered.

The first theory holds that anatomical alterations, subtle but crucial, have been and are occurring regularly in the human brain. In this postulation anatomical alterations underlie the brain capability needed for current technological and intellectual gains. In this theory the failure to develop the current technological wonders in previous centuries was due to inadequate brain development. Little or no evidence from either comparative neuroanatomy or behavioral studies supports such a theory. The behavioral (intellectual and technical) changes currently occurring are of such magnitude and have taken place with such rapidity that alterations in anatomical structures crucial to these changes appear implausible.

A second theory posits no real change in brain anatomy. Rather, cortical structures, particularly those novel in the human brain, provide relatively virgin territory with as yet

\section{EXECUTIVE FUNCTION}

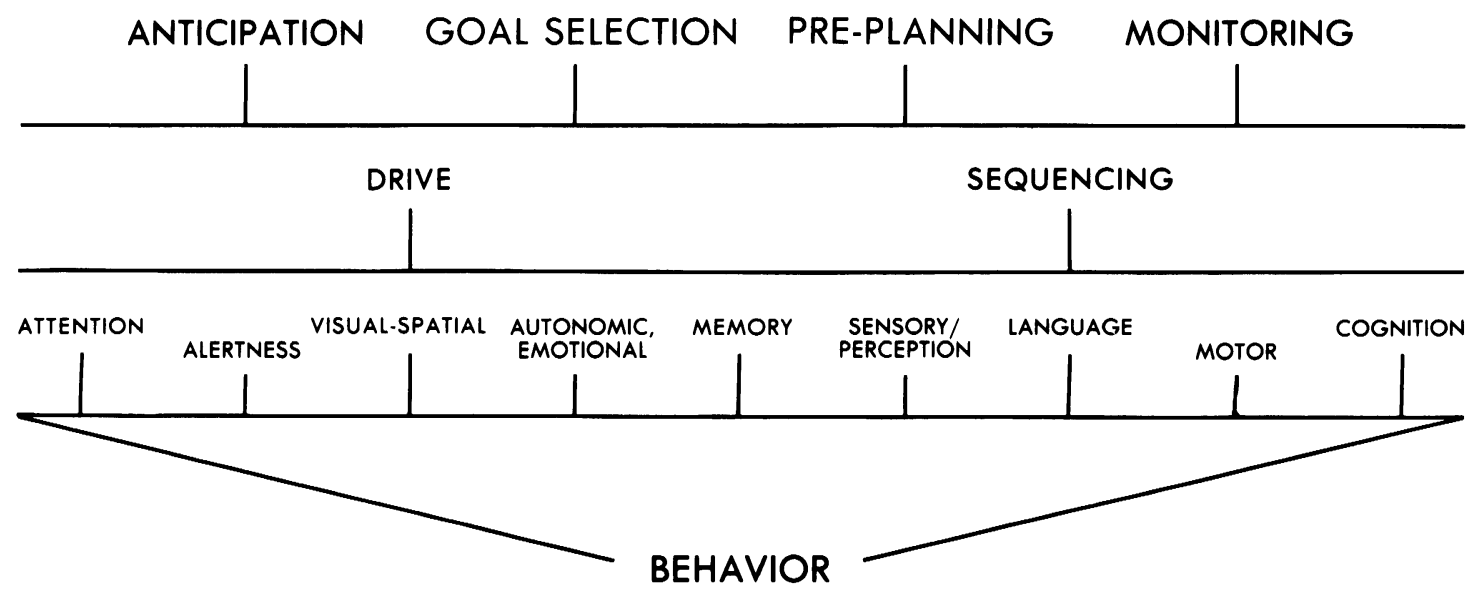

FIG. 2. Diagram to illustrate the hierarchical status of higher cognitive functions. (From Stuss and Benson, 1986, with permission.) 
(a)

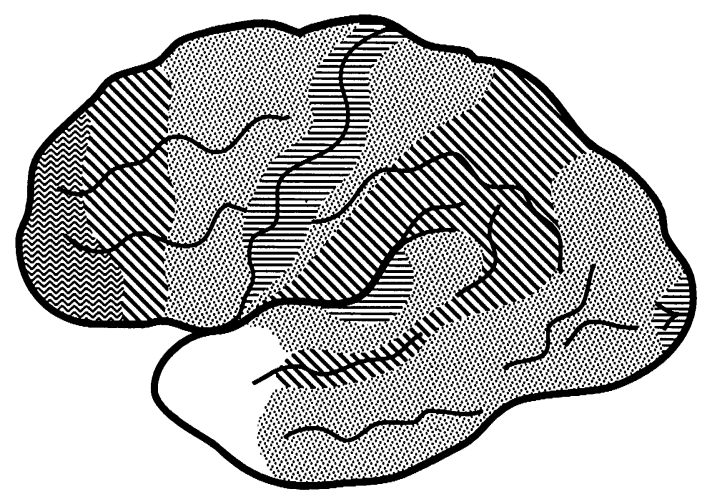

(b)

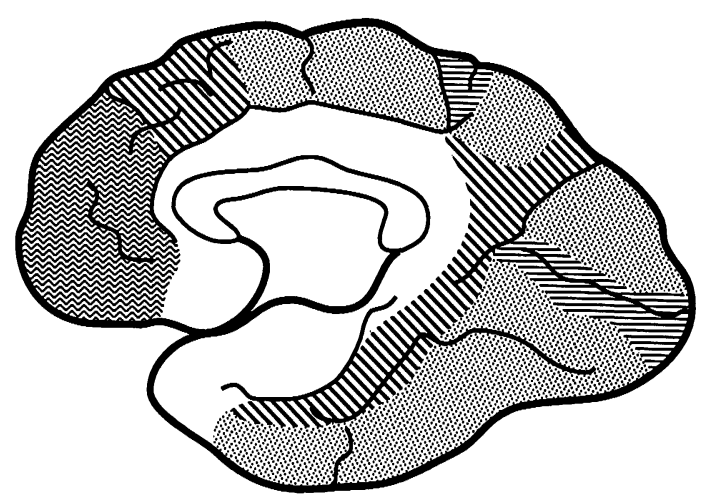

(c)

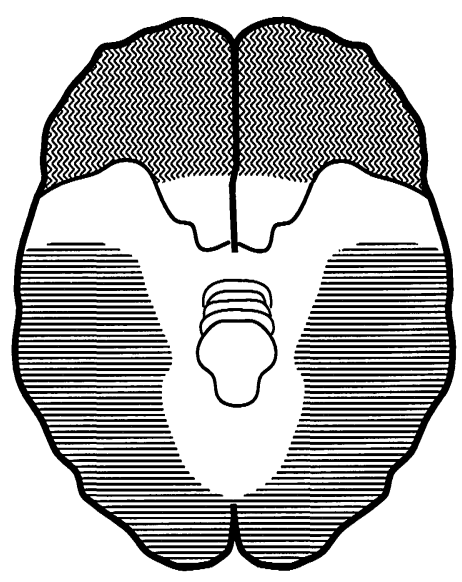

FIG. 3. Three diagrammatic views of cortex [(a) lateral; (b) sagittal; (c) inferior] with four areas of cortex delineatedprimary (isotypic) cortex = light lines; unimodal association cortex = dotted matrix; heteromodal association cortex = heavy diagonal lines; supramodal association cortex = horizontal wavy bands.

unexploited possibilities. By this postulate, the explosion of technological and intellectual information has been possible, at least on a neuroanatomical structure basis, for many millennia and the ongoing accomplishments are based only on accumulated information.

A third theory suggests that subtle alterations in the hierarchical structure of higher cortical functions in the human brain allow behavioral evolution. That significant differences in key areas of cortex, particularly prefrontal, distinguish humans from other species is accepted. That some of these differences are of recent origin, possibly ongoing, can neither be proved nor refuted. To investigate this postulation, analysis of the anatomical-behavioral hierarchy is indicated.
Based on clinical experience abetted by anatomical and physiological evidence, functional divisions of human cortex have been suggested (Mesulam, 1985; Stuss and Benson, 1986). Four cortical areas, distinguished by anatomical structure and/or functional qualities, have been suggested: (1) primary (isotypic) cortex; (2) unimodal association cortex; (3) heteromodal association cortex; (4) supramodal association cortex (Benson, 1993). Figure 3 outlines general boundaries for the four areas of cortex on line drawings of human brain surfaces.

Primary (isotypic) cortex is the cortical area that receives (or sends) stimuli connecting the brain to external sources. Three major sensory regions are the primary visual cortex in the calcarine region of the occipital lobes, the 
primary auditory cortex in the superior surfaces of the temporal lobes, and the primary somesthetic cortex in the immediate post-Rolandic region. Just anterior to the fissure of Rolando lies the primary motor cortex. These regions provide the neural linkage that connects brain cortex to the physical environment.

Immediately surrounding the four regions of primary cortex are relatively vast areas of unimodal association cortex. Each of these brain areas is dedicated to processing stimuli from a single sensory source. Here the raw materials provided through primary cortex are distinguished, compared and patterned to form a percept, but only in a single modality. The step of unimodal perception is essential to higher mental functioning-all knowledge of the real world stems from sensing and perceiving.

Intermixed with the unimodal association cortex regions are two areas of heteromodal association cortex. It is here that unimodal percepts are associated with information from other sensory modalities and from prior experiences (memories). Most simple cross-modal associations occur in the inferior parietal cortex. However, many, probably most, high level intellectual functions demand serial processing, the maintenance of information in appropriate sequence over time, a process that demands prefrontal activity (Fuster, 1980; Stuss and Benson, 1986; Goldman-Rakic, 1990). Both the parietal and the prefrontal heteromodal association cortex regions have rich connections to unimodal association cortex. This consortium of neural structure forms the complex networks necessary to form the distributed mental processes necessary for higher cortical functioning (Goldman-Rakic, 1990). Heteromodal association cortex is relatively recent in the phylogenetic development. While some heteromodal association cortex is present in higher apes, it has neither the large size nor the rich connections that characterize human heteromodal association cortex. High level cognitive activities such as language, visual imagery, cognitive manipulation and others are performed in selected (and dedicated) neural processing units as demonstrated by the relatively limited dysfunction (e.g., aphasia, acalculia, agnosia) produced by focal lesions in heteromodal association cortex.

A fourth cortical area, supramodal association cortex (Benson, 1993), lies rostral in the brain. Little or no supramodal association cortex is present in other animals, even higher apes. Observations of behavioral disorders following damage to supramodal association cortex suggest that this area monitors and judges the activities of the cognitive networks and selects preferred responses that are influenced, to a considerable extent, by limbic (internal) input. The supramodal association cortex apparently directs activities that demand novel, non-reflex responses (Pribram, 1973; Shallice and Evans, 1978). The function of supramodal association cortex in the evolution of the brain

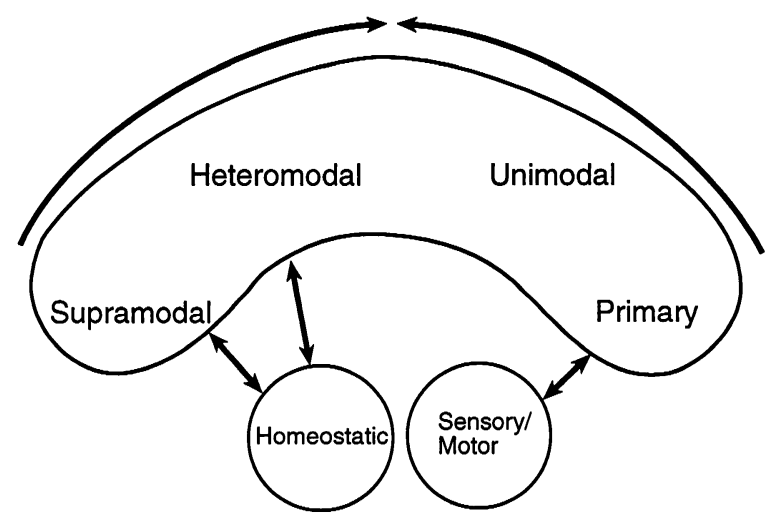

FIG. 4. Graphic illustration demonstrating bimodal subcorticalcortical connections and general directions of sensory-motor and homeostatic influence in the cortex.

has been accompanied by marked improvements in control (management) of brain functions.

All areas of cortex do not receive equal quantities or types of subcortical neural input. In fact, subcortical input is both limited and specific. Figure 4 presents a stylized view of subcortical-cortical connections. Sensory-motor stimuli reach the cortex via primary cortex; relatively little sensory-motor input travels directly to unimodal, heteromodal or supramodal association areas. In contrast, supramodal and, to a lesser extent, anterior heteromodal association cortices receive considerable input from limbic and subcortical motor structures. The four major areas of cortex are richly interconnected by cortical-cortical fibers with a basic caudal-rostral flow of sensory-motor information and a reverse direction for homeostatic influences.

\section{IMPLICATIONS}

Whether the progression in human intellectual competency, so notable through the past several centuries, is based on subtle neuroanatomical alterations, a constantly enlarging database, or both, remains conjectural. Either scenario would suggest that continuing advancement is probable. If anatomical alteration actually underlies the rapid technological changes in manufacture that characterize 20th century human behavior, similar advances could be anticipated for the future. On the other hand, if the obvious advances in behavior have occurred without brain alteration, it implies that the human brain has long been capable of the technological advances only now appearing and there is no indication that all potential achievements have already occurred. With either explanation, more and better technological accomplishments can be anticipated.

Thus, continuing alterations of human behavior, particularly those based on management and manipulation of higher level cognitive activity, appear probable. It is even possible that human moral and esthetic competencies, cur- 
rently showing less tendency to advance than the technological spheres, may also undergo change. The human brain, as currently constituted and with the possibility of future anatomical alterations, may be capable of creating a truly "kinder and gentler" world.

\section{REFERENCES}

Benson D (1993) Progressive frontal dysfunction. In: Dementia (Ed. A Brun). Karger, Basel, in press.

Bianchi L (1895) The functions of the frontal lobes. Brain, 18, 497-522.

Blinkov SM and Glezer II (1968) The Human Brain in Figures and Tables. Plenum Press, New York.

Brodmann K (1912) Neue Ergebnisse über die vergleichende histologische Lokalisation der gross-hirnrinde mit besenderer Berucksichtingung des Stirnhirns. Anatomischer Anzeiger (Supp.), 41, 157-216.

Campbell CBG (1975) The central nervous system: Its uses and limitations in assessing phylogenetic relationships. In: Phylogeny of the Primates (Eds WP Luckett and FS Szalay), pp. 183-197. Plenum Press, New York.

Chapman LF and Wolff HG (1959) The cerebral hemispheres and the highest integrative functions of man. Archives of Neurology, 1, 357-424.

Cook ND (1986) The Brain Code: Mechanisms of Information Transfer and the Role of the Corpus Callosum. Methuen, New York.

Damasio A (1985) The frontal lobes. In: Clinical Neuropsychology (Eds KM Heilman and E Valenstein), pp. 339-376. Oxford University Press, New York.

Feuchtwanger E (1923) Die funktionen des Stirnhirns ihre pathologie und psychologie. Monographien aus dem Gesamtgebiete der Neurologie und Psychiatrie, (Berlin), Vol. 38, pp. $1-193$.

Franz SI (1907) On the Functions of the Cerebrum of the Frontal Lobes. The Science Press, New York.

Fulton JF (1952) The Frontal Lobes and Human Behavior. Charles C. Thomas, Springfield, IL.

Fuster JM (1980) The Prefrontal Cortex: Anatomy, Physiology and Neuropsychology of the Frontal Lobe. Raven Press, New York.

Fuster JM (1989) The Prefrontal Cortex: Anatomy, Physiology and Neuropsychology of the Frontal Lobe, 2nd edn. Raven Press, New York.

Goldberg E and Bilder RM Jr (1987) The frontal lobes and hierarchical organization of cognitive control. In: The Frontal Lobes Revisited (Ed. E Perecman), pp. 159-187. IRBN Press, New York.

Goldman-Rakic PS (1990) Cellular and circuit basis of working memory in prefrontal cortex of nonhuman primates. In: Progress in Brain Research, Vol. 85, pp. 325-336. Elsevier, Amsterdam.

Goldstein K (1944) Mental changes due to frontal lobe damage. Journal of Psychology, 17, 187-208.

Grünbaum ASF and Sherrington CS (1903) Observations on the physiology of the cerebral cortex of the anthropoid apes. Proceedings of the Royal Society, London, 72, 152-155.

Halstead W (1947) Brain and Intelligence: A Quantitative Study of the Frontal Lobes. University of Chicago Press, Chicago,

Hebb DO (1945) Man's frontal lobes. Archives of Neurology and Psychiatry, 54, 10-24.
Hodos W (1970) Evolutionary interpretation of neural and behavioral studies in living vertebrates. In: The Neurosciences: Second Study Program (Ed. FO Schmidt), pp. 26-39. Rockefeller Univ. Press, New York.

Jerison HJ (1991) Brain Size and the Evolution of Mind. American Museum of Natural History, New York.

Kotchetkova VI (1978) Paleoneurology. Winston/Wiley, Washington, DC.

Lashley KS (1929) Brain Mechanisms and Language. University of Chicago Press, Chicago.

Luria AR (1973) The Working Brain (transl. B Haigh). Basic Books, New York.

Mesulam M-M (1985) Patterns in behavioral neuroanatomy: Association areas, the limbic system and hemispheric specialization. In: Principles of Behavioral Neurology (Ed. M-M Mesulam), pp. 1-70. Davis, Philadelphia.

Mettler FA (Ed.) (1949) Columbia-Greystone Associates: Selective Partial Ablation of the Frontal Cortex: A Correlative Study of its Effects on Human Psychotic Subjects. Hoeber, New York.

Miller GA, Galanter EH and Pribram KH (1960) Plans and the Structure of Behavior. Holt, Rinehart and Winston, New York.

Munk H (1890) Ueber die Functionen der Grosshirnrinde. Hirschwald, Berlin.

Nauta WJH (1971) The problem of the frontal lobe: A reinterpretation. Journal of Psychiatric Research, 8, 167-187.

Pandya DN, Van Hoesen GW and Mesulam M-M (1981) Efferent connections of the cingulate gyrus in the rhesus monkey. Experimental Brain Research, 42, 319-330.

Pribram KH (1960) The intrinsic systems of the forebrain. In: Handbook of Physiology, Vol. II: Neurophysiology (Eds J Field, HW Magoun and VE Hall), pp. 1323-1344. American Physiological Society, Washington, DC.

Pribram KH (1973) The primate frontal cortex, executive of the brain. In: Psychophysiology of the Frontal Lobes (Eds KH Pribram and AR Luria), pp. 293-314. Academic Press, New York.

Rylander G (1939) Personality Changes After Operations on the Frontal Lobes: A Clinical Study of 32 Cases. Munksgaard, Copenhagen.

Shallice T (1982) Specific impairments of planning. In: The Neuropsychology of Cognitive Function (Eds DE Broadbent and L Weiskrantz), pp. 199-209. The Royal Society, London.

Shallice T and Evans ME (1978) The involvement of the frontal lobes in cognitive estimation. Cortex, 4, 294-303.

Stuss DT and Benson DF (1986) The Frontal Lobes. Raven Press, New York.

Teuber H-L (1959) Some alterations in behavior after cerebral lesions in man. In: Evolution of Nervous Control From Primitive Organisms to Man (Ed. AD Bass), pp. 157-194. American Association for the Advancement of Science, Washington, DC.

Teuber H-L (1964) The riddle of frontal lobe function in man. In: The Frontal Granular Cortex and Behavior (Eds JM Warren and K Akert), pp. 410-444. McGraw-Hill, New York.

Tilney F (1928) The Brain, From Ape to Man. Hoeber, New York.

Weinstein S and Teuber HL (1957) Effects of penetrating brain injury on intelligence test scores. Science, 125, 1036-1037.

Young JZ (1987) Philosophy and the Brain. Oxford University Press, Oxford.

Young RM (1970/1990) Mind, Brain and Adaptation in the Nineteenth Century. Oxford University Press, New York. 


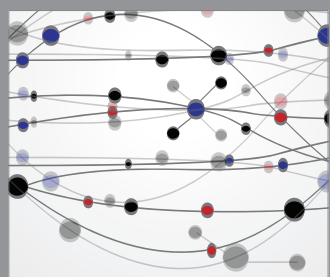

The Scientific World Journal
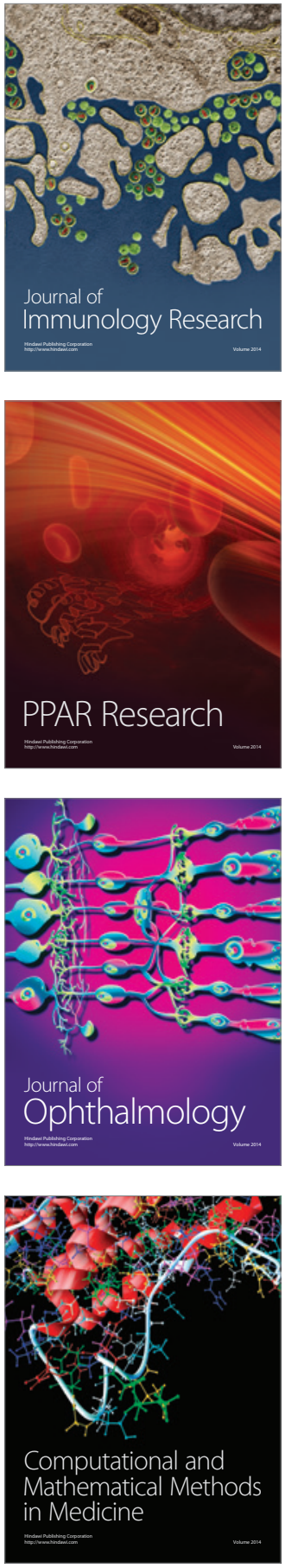

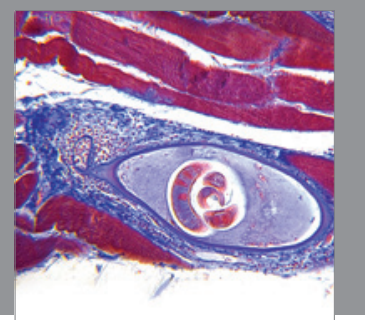

Gastroenterology

Research and Practice
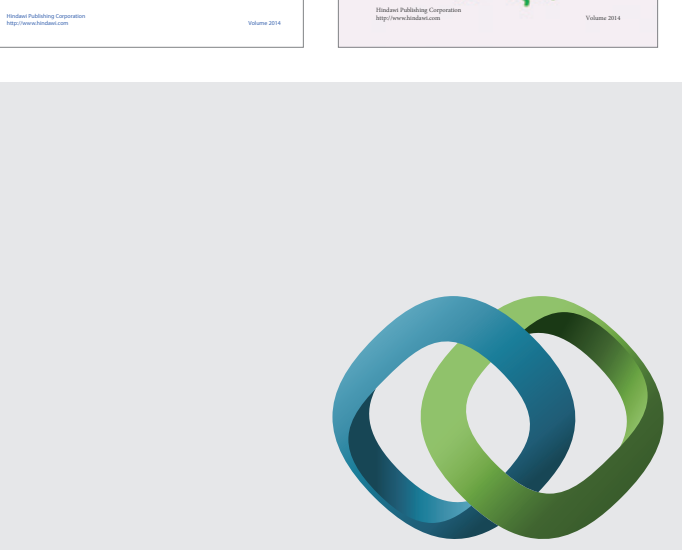

\section{Hindawi}

Submit your manuscripts at

http://www.hindawi.com
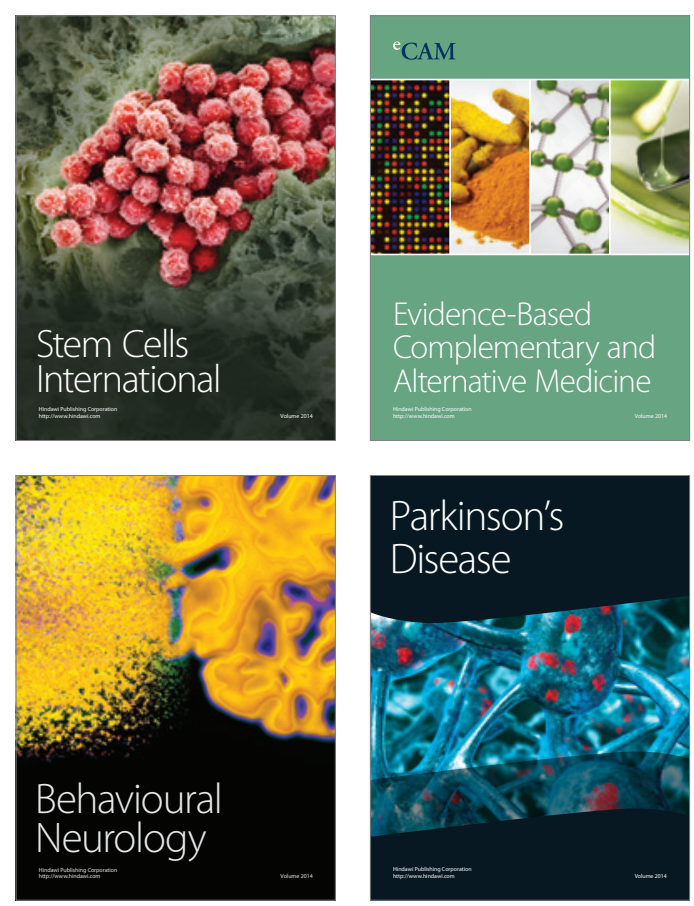

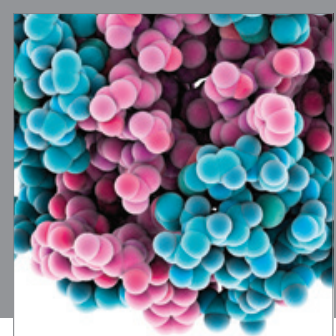

Journal of
Diabetes Research

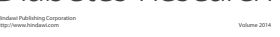

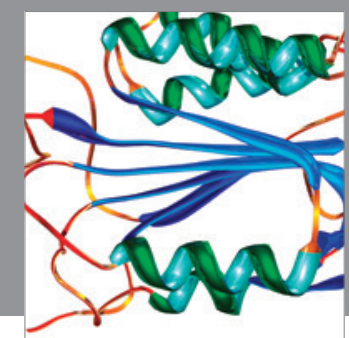

Disease Markers
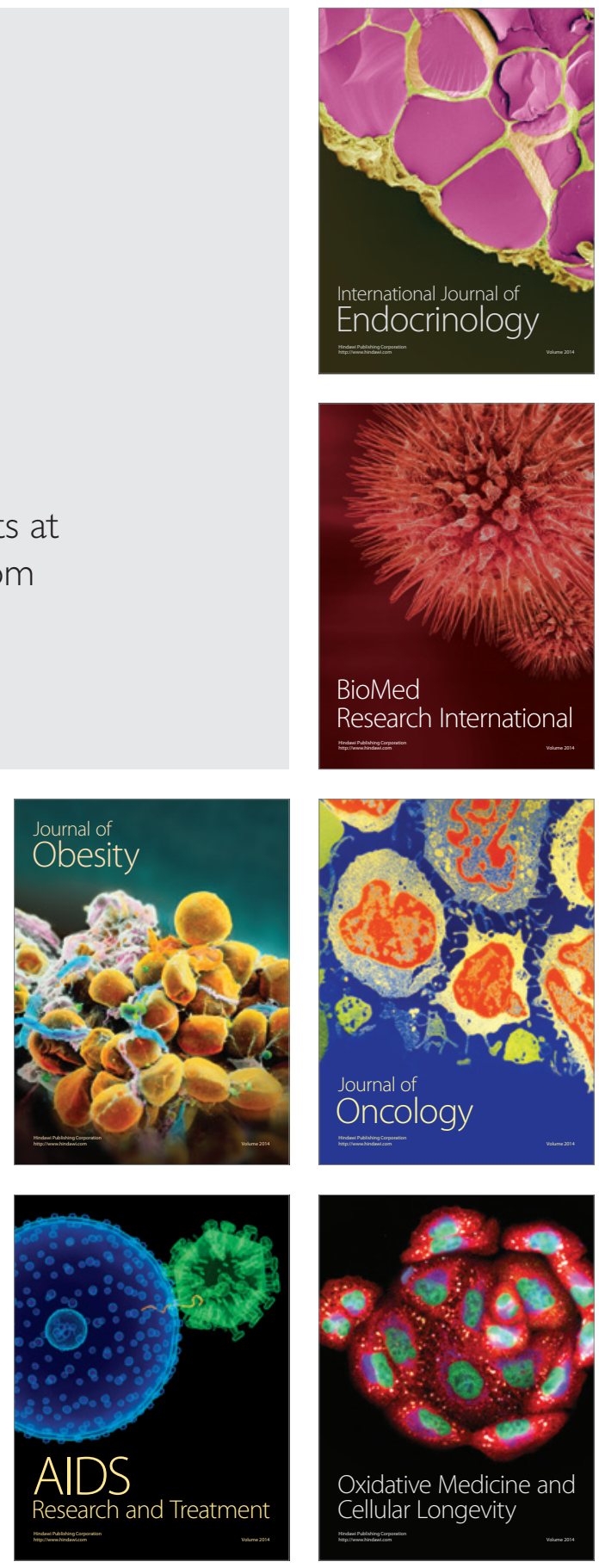